Ann. Biol. anim. Bioch. Biophys., I968, 8 (4), 581-583.

\title{
ÉTUDE DE LA VIDANGE STOMACALE DU LAIT ENTIER CHEZ LE VEAU PRERUMINANT
}

\author{
C.-M. MATHIEU \\ Station de Recherches sur l'Élevage des Ruminants, \\ Centre de Recherches zootechniques et vétérinaires sur les Ruminants, \\ 63 - Theix près Clermont-Ferrand \\ Institut national de la Recherche agronomique
}

La vidange stomacale du lait semble jouer un 'rôle très important chez le veau préruminant, tant dans la cinétique et l'efficacité de la digestion que dans l'apparition des troubles digestifs (diarrhée).

Nous avons donc cherché à connaître le débit et la composition du contenu digestif à la sortie de la caillette. La pose de deux canules duodénales permanentes (Asr, I962) s'étant révélée délicate, nous avons mis au point une canule en polyvinyle constituée d'un canal central (lumière de $13 \mathrm{~mm}$ ) à partir duquel partent perpendiculairement deux canaux latéraux contigus (lumière de II mm chacun). Le canal central permet un écoulement normal du contenu digestif ; l'adjonction d'une lamelle entre les deux canaux latéraux obstrue la lumière centrale, le contenu digestif s'écoule alors par un canal latéral et est réintroduit simultanément à l'aide d'une pompe dans l'autre canal latéral.

Nous avons posé une telle canule à l'entrée du duodénum sur 3 veaux âgés d'environ 3 semaines. Nous avons recueilli le contenu digestif correspondant à 9 repas de lait entier (taux butyreux moyen de $30 \mathrm{p}$. I ooo). Le prélèvement durait $7 \mathrm{~h}$ au cours duquel nous recueillions 9 échantillons de $50 \mathrm{~g}$. Le tableau I rassemble les valeurs moyennes du débit et de la composition des échantillons.

Le débit est maximum dès la fin du repas, puis il diminue progressivement pour atteindre, au bout de $7 \mathrm{~h}$, une valeur voisine de celle observée avant le repas. La durée moyenne au cours de laquelle la quantité du contenu digestif recueilli correspond à la quantité de lait ingéré est d'environ $7 \mathrm{~h}$. Le $\mathrm{pH}$ est maximum après le repas puis diminue constamment.

La teneur en matière sèche du contenu digestif est relativement constante (6 à 7 p. IOo). Sa teneur en lactose et en minéraux augmente rapidement après le repas, puis diminue pour atteindre, $7 \mathrm{~h}$ plus tard, une valeur trois fois plus faible. En revanche, les teneurs en matières azotées et surtout en matières grasses du contenu digestif sont faibles après le repas ; elles augmentent constamment ensuite jusqu'à 


\begin{tabular}{|c|c|c|c|c|c|c|c|c|c|}
\hline \multirow{12}{*}{ 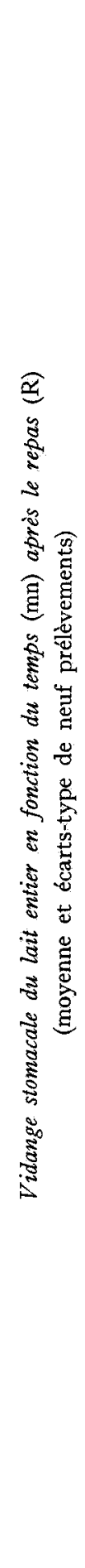 } & $\begin{array}{l}\text { है } \\
\text { + } \\
+ \\
\simeq\end{array}$ & $\begin{array}{l}20 \\
m^{2} \\
H \\
0 \\
0\end{array}$ & $\mid \begin{array}{l}0 \\
0 \\
0 \\
H \\
0 \\
0 \\
0\end{array}$ & $\begin{array}{l}\stackrel{\circ}{\rightarrow} \\
+1 \\
\text { Ho } \\
\text { oi }\end{array}$ & $\begin{array}{l}\overrightarrow{a i} \\
+1 \\
\approx \\
\infty\end{array}$ & 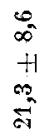 & $\begin{array}{l}5 \\
+1 \\
0 \\
0\end{array}$ & 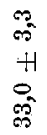 & $\begin{array}{l}22 \\
0 \\
+1 \\
+1 \\
\text { an } \\
5\end{array}$ \\
\hline & $\begin{array}{l}\stackrel{\infty}{\infty} \\
+ \\
\sim\end{array}$ & 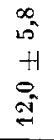 & $\begin{array}{l}10 \\
0 \\
+1 \\
0 \\
0 \\
0\end{array}$ & $\begin{array}{l}g \\
0 \\
+1 \\
\infty \\
o \\
\text { of }\end{array}$ & $\begin{array}{l}\infty \\
0 \\
+1 \\
\sigma \\
r\end{array}$ & \begin{tabular}{l}
$\infty$ \\
$\infty$ \\
+1 \\
+1 \\
$=$ \\
\multirow{4}{*}{+}
\end{tabular} & $\begin{array}{l}\stackrel{3}{2} \\
+1 \\
5 \\
5\end{array}$ & $\begin{array}{l}\text { s. } \\
\text { s. } \\
H \\
\text { os } \\
\text { A }\end{array}$ & $\begin{array}{l}12 \\
+1 \\
+1 \\
8 \\
8\end{array}$ \\
\hline & $\begin{array}{l}\stackrel{8}{\sim} \\
+ \\
4\end{array}$ & 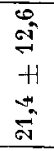 & $\begin{array}{l}\sigma \\
8 \\
+1 \\
+ \\
-\end{array}$ & $\begin{array}{l}\vec{g} \\
\dot{\sigma} \\
H \\
\text { H } \\
\sigma_{0}\end{array}$ & $\begin{array}{l}a^{\prime} \\
+1 \\
\infty \\
0\end{array}$ & $\begin{array}{l}12 \\
\text { on } \\
+1 \\
5 \\
5 \\
6\end{array}$ & $\begin{array}{l}\Rightarrow \\
+ \\
\text { O } \\
\therefore\end{array}$ & $\begin{array}{l}\infty \\
\stackrel{\infty}{0} \\
+ \\
\stackrel{H}{2}\end{array}$ & $\begin{array}{l}0 \\
\text { o } \\
H \\
0 \\
0 \\
0\end{array}$ \\
\hline & $\begin{array}{l}8 \\
+ \\
\curvearrowleft\end{array}$ & $\begin{array}{l}+1 \\
0 \\
+1 \\
\overrightarrow{0}\end{array}$ & \begin{tabular}{l}
$\approx$ \\
\multirow{2}{*}{} \\
+ \\
$=$ \\
$=$
\end{tabular} & $\begin{array}{l}0^{\infty} \\
-1 \\
-1 \\
0^{-1}\end{array}$ & $\begin{array}{l}+ \\
0 \\
+1 \\
0 \\
0\end{array}$ & 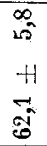 & $\begin{array}{l}5 \\
= \\
+1 \\
0 \\
0\end{array}$ & $\begin{array}{l}\infty \\
+ \\
+1 \\
\infty \\
\stackrel{\infty}{\circ}\end{array}$ & 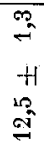 \\
\hline & $\begin{array}{l}8 \\
+ \\
\not\end{array}$ & 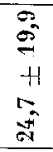 & $\begin{array}{l}\text { a } \\
+ \\
+1 \\
0 \\
\text { oi }\end{array}$ & $\begin{array}{l}\stackrel{+}{0} \\
+1 \\
+ \\
n^{2}\end{array}$ & $\begin{array}{l}m_{x} \\
\sigma^{2} \\
+1 \\
\tilde{\sigma}\end{array}$ & 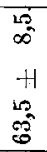 & $\begin{array}{l}\stackrel{6}{-} \\
+1 \\
0 \\
0\end{array}$ & $\begin{array}{l}\stackrel{m}{\rightarrow} \\
H \\
\text { H. } \\
\stackrel{-}{*}\end{array}$ & $\begin{array}{l}+ \\
+1 \\
+ \\
=\end{array}$ \\
\hline & $\begin{array}{l}9 \\
+ \\
+ \\
\alpha\end{array}$ &  &  & $\begin{array}{l}0 \\
0 \\
H \\
0 \\
0 \\
0\end{array}$ & $\begin{array}{l}10 \\
8 \\
+1 \\
0 \\
0\end{array}$ & $\begin{array}{l}+ \\
\infty \\
+1 \\
\infty \\
0\end{array}$ & $\begin{array}{l}\stackrel{\infty}{0}_{-} \\
H \\
+1 \\
\infty \\
\infty \\
\infty\end{array}$ & $\begin{array}{l}0 \\
\text { oi } \\
H \\
H \\
\infty \\
\infty \\
\infty\end{array}$ & $\begin{array}{l}\text { on } \\
\text { s } \\
11 \\
0 \\
0 \\
0\end{array}$ \\
\hline & $\begin{array}{l}\text { 오 } \\
+ \\
x\end{array}$ & 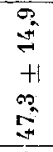 & $\begin{array}{l}\stackrel{O}{-} \\
+1 \\
-5\end{array}$ & $\begin{array}{l}3 \\
0 \\
+1 \\
o \\
*\end{array}$ & $\begin{array}{l}\text { s } \\
+1 \\
+1 \\
+\infty\end{array}$ & $\begin{array}{l}\infty \\
\infty \\
+1 \\
\vdots \\
0\end{array}$ & $\begin{array}{l}\stackrel{9}{-} \\
+1 \\
=\end{array}$ & $\begin{array}{l}5 \\
+1 \\
+1 \\
\infty \\
\infty\end{array}$ & $\begin{array}{l}10 \\
+1 \\
+1 \\
+2 \\
0\end{array}$ \\
\hline & $\begin{array}{l}9 \\
+ \\
\infty\end{array}$ & 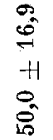 & $\mid \begin{array}{c}30 \\
-1 \\
+1 \\
6 \\
0\end{array}$ & $\begin{array}{l}2 \\
+1 \\
+2 \\
+2\end{array}$ & $\begin{array}{l}\infty \\
0 \\
H \\
0 \\
N\end{array}$ & 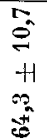 & $\begin{array}{l}23 \\
+1 \\
+1 \\
\infty \\
\infty\end{array}$ & 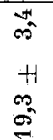 & $\begin{array}{l}\theta \\
+ \\
H \\
+1 \\
0\end{array}$ \\
\hline & $\underset{1}{2}$ & 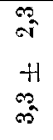 & $\begin{array}{l}\tilde{\sigma} \\
0 \\
+1 \\
\text { on } \\
0\end{array}$ & $\begin{array}{l}0 \\
0 \\
+1 \\
\text { a } \\
\text { a }\end{array}$ & $\begin{array}{l}9 \\
+ \\
+1 \\
0 \\
\text { a }\end{array}$ & $\begin{array}{l}5 \\
0 \\
+1 \\
?\end{array}$ & $\begin{array}{l}\text { कू } \\
+ \\
H \\
+ \\
0\end{array}$ & $\begin{array}{l}6 \\
\infty \\
+1 \\
\overrightarrow{00}\end{array}$ & $\begin{array}{l}0 \\
+1 \\
+3\end{array}$ \\
\hline & 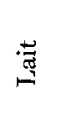 & & & & $\begin{array}{l}5 \\
0 \\
+1 \\
+4 \\
o n \\
g\end{array}$ & $\begin{array}{l}0 \\
= \\
+1 \\
\overrightarrow{\mathrm{i}}\end{array}$ & $\begin{array}{l}5 \\
0 \\
H \\
\infty \\
20\end{array}$ & 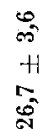 & $\begin{array}{l}\text { an } \\
\text { is } \\
+1 \\
\tilde{N}\end{array}$ \\
\hline & &  & 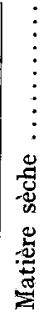 & & 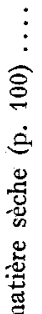 & 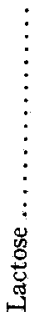 &  & 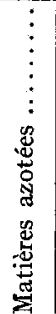 & 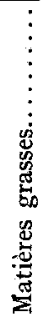 \\
\hline & (uw & Ұ!qश & & İ⿴囗十் &  & & & & \\
\hline
\end{tabular}


des valeurs respectivement 3 fois et 5 fois plus élevées. En conséquence, les quantités de lactose, de matière sèche, de matières azotées et de matières grasses, entrant dans le duodénum dans les $7 \mathrm{~h}$ suivant l'ingestion, ont été égales, respectivement à $72 \mathrm{p}$. 100, 6I p. 100, $57 \mathrm{p}$. 100 et $5 \mathrm{I} \mathrm{p}$. Ioo des quantités ingérées.

Ces résultats sont dans l'ensemble en accord avec ceux que MYLREA (I966 a et $b$ ) a obtenu sur des veaux porteurs de 2 canules séparées (canule de prélèvement et canule de réintroduction).

En utilisant la méthode de ROWLAND améliorée par ASChAFFENBURG et DREWRY (1959), nous avons dosé différentes fractions azotées sur les trois échantillons prélevés à $\mathrm{R}+40 \mathrm{mn}, \mathrm{R}+\mathrm{r} 20 \mathrm{mn}, \mathrm{R}+420 \mathrm{mn}$. La proportion de l'azote non protéique a été relativement constante $(48,3$ p. Ioo $\pm 6,5$ de l'azote total), alors que la proportion de 1'azote de la caséine augmentait avec le temps (I2,5, 19,7 et 22,8 p. 10o).

Comparés à la composition du lait, ces résultats mettent en évidence l'importance de la digestion dans la caillette et notamment de la coagulation sur l'évolution de la composition du contenu digestif à l'entrée du duodénum. Dans une première phase, correspondant à l'expulsion du lactosérum, le contenu est riche en lactose et en minéraux ; par la suite, la lyse du coagulum enrichit le contenu digestif en matières azotées et en matières grasses. Parmi les matières azotées, une grande partie de la caséine ( 85 p. Ioo environ), est dégradée dans la caillette.

Reçu pour publication en juillet 1968

\section{SUMMARY}

GASTRIC EMPTYING IN YOUNG CALVES FED WHOLE MILK

Gastric emptying of 9 meals of whole milk was studied on 3 duodenal-canulated calves. The flow reached a maximum rapidly after feeding, and then decreased with a return to pre-feeding level at $7 \mathrm{hr}$. The concentration of lactose and minerals rised just after feeding, then there was a continuous increase in nitrogen and lipid concentrations.

\section{RÉFÉRENCES BIBLIOGRAPHIQUES}

Aschaffendurg R., DRewry J., 1959. Routine estimation of non-casein proteins of milk. XV Int. Dairy Congr., 8, I63i-1637.

AsH R. W., I962. Gastro-intestinal re-entrant cannulea for studies of digestion in sheep. Anim. Prod., 4, 309-3 2 .

MYLREA P. J., 1966. Digestion of milk in young calves. I. Flow and acidity of the contents of the small intestine. Res. Vet. Sci., 7,333 -341.

MYLREA P. J., I966. Digestion of milk in young calves. II. The absorption of nutrients from the small intestine. Res. Vet. Sci., 7, 394-406. 